\title{
La acreditación de idiomas en las universidades españolas: Un estudio cuantitativo en la Universidad de Córdoba.
}

\section{Language certification in the Spanish Universities: A quantitative study at the University of Córdoba}

\author{
Antonio Raigón Rodríguez. \\ Universidad de Córdoba (España). \\ antonio.raigon@uco.es \\ Ángela Larrea Espinar. \\ Universidad de Córdoba (España). \\ angela.larrea@uco.es
}

\section{RESUMEN.}

Con la implantación casi generalizada de requisitos lingüísticos en los grados de las universidades españolas, la acreditación de los niveles de competencia en lenguas extranjeras ha tomado una relevancia esencial. Dentro de este trabajo, presentaremos una visión global del estado de la cuestión en España, para más tarde centrarnos en el análisis de la acreditación lingüística del alumnado de la Universidad de Córdoba. Nuestro estudio estadístico, basado en un grupo de más de dos mil alumnos, nos proporcionará datos con los que dibujar un panorama aproximado del nivel de acreditación idiomática en nuestra universidad, teniendo en cuenta variables tales como la nota de acceso, la macroárea, el sexo o el idioma seleccionado para cumplir con el requisito a la hora de egresar. Asimismo, podremos dilucidar si caminamos hacia una Europa más plurilingüe o no.

\section{PALABRAS CLAVE.}

Aprendizaje de idiomas, Acreditación de idiomas, B1, Enseñanza Superior.

\section{ABSTRACT.}

With the widespread arrival of language competence requirements at the Spanish university degrees, language certification has gained an essential relevance. In this study, we will present firstly a state of the matter, to later focus on our analysis on the certification process of the University of Cordoba's students. Our statistical study, based on a sample of more than two thousand students, will provide enough data to sketch a close outlook on how students comply with this requirement, taking into account variables such as entry mark, macroarea, sex and language chosen. Moreover, we will be able to determine whether this measure is helping us build a plurilingual Europe.

\section{KEY WORDS.}

Language learning, language certification, B1, Higher Education.

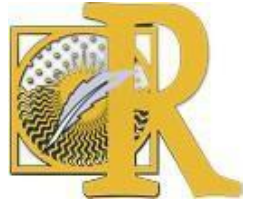




\section{Introducción.}

La llegada del Espacio Europeo de Educación Superior (EEES) y de los nuevos grados trajeron consigo la implantación casi generalizada de requisitos lingüísticos para el alumnado tanto para egresar como para acceder a determinados másteres. A efectos prácticos, actualmente, casi la totalidad del alumnado universitario en el sistema español tiene que acreditar mediante un certificado su capacitación en una segunda lengua.

Además de ser un requisito para egresar, las acreditaciones lingüísticas son necesarias para otros ámbitos de la vida profesional y universitaria. Más que un fin, las acreditaciones son el resultado de un proceso de aprendizaje que queda reflejado de manera formal en el certificado que emiten las diferentes instituciones, públicas y privadas, reconocidas por las Universidades a estos efectos.

El requisito lingüístico supone una barrera para el alumnado a la hora de obtener su título de grado. Esta traba, que no debiera ser tal ya que no se trata de un nivel elevado (por lo general, el B1), tiene su origen en el desigual desarrollo de competencias de los alumnos españoles en las clases de idiomas de educación primaria y secundaria. Según el último Eurobarómetro disponible (2012: 15), el 54\% de los europeos puede mantener una conversación en una lengua extranjera con normalidad. España se sitúa en el puesto 22 de 27 , con un $46 \%$, habiendo subido dos puntos porcentuales los resultados, que eran más pobres en el anterior estudio (2005).

Pasada casi una década de la llegada de los nuevos grados, este estudio pretende analizar cómo está cumpliendo el alumnado de la UCO con el requisito lingüístico para egresar, sus niveles de acreditación lingüística y la relación entre diferentes variables y dicho nivel.

\section{Marco teórico.}

\subsection{La publicación del Marco y los niveles de acreditación lingüística.}

A principios de los años 70 se desarrolló la idea del Threshold level (en español, nivel umbral), conocido posteriormente como B1 (Council of Europe, 1975). En años posteriores se fueron incorporando y revisando niveles y ya en 1991, un simposio intergubernamental en Rüschlikon recomendó al Consejo de Europa (Little, 2011) elaborar un Marco Común Europeo de Referencia para las Lenguas (MCERL) "a Common European Framework of Reference for language learning at all levels" (Council of Europe, 1992: 37).

Aunque el texto en español se publicó en el 2002, el MCERL vio la luz en inglés y en francés en 2001, el año Europeo de las Lenguas; fue creado para facilitar la cooperación entre las instituciones educativas de los distintos países, promoviendo la transparencia y coherencia en materia de aprendizaje de lenguas. Además, el Marco proporciona una base común para el reconocimiento de certificaciones lingüísticas estableciendo unos niveles de referencia comunes que miden el dominio de todas las lenguas. Los niveles de referencia recogen lo que los estudiantes tienen que conseguir en las distintas etapas del aprendizaje (Clouet, 2010).

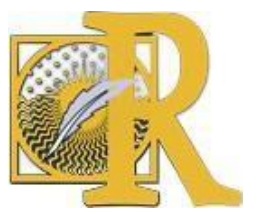


Esta base común puede también proporcionar unidad en cursos o programas de aprendizaje de idiomas, calificaciones, exámenes, libros de texto, y planes de estudio en Europa (Broek y Van den Ende, 2013).

En líneas generales, el Marco establece 6 niveles comunes de referencia que van desde el A1 al C2 y describe competencias generales y competencias comunicativas. Asimismo, se centra positivamente en lo que el hablante/aprendiz puede hacer en otro idioma mediante

54 escalas ilustrativas que contienen descriptores de cada nivel para cada destreza o habilidad (34 para actividades comunicativas, 7 para estrategias afines y 13 para aspectos cualitativos del dominio comunicativo de la lengua -North, 2007). Como hemos mencionado anteriormente, estos descriptores no se asocian a ninguna lengua en concreto, sino que, en teoría, sirven para cualquier lengua.

Desde el punto de vista de Alderson (2007), lo más relevante del MCERL es la dimensión horizontal, esto es, el esquema descriptivo; sin embargo, han sido las escalas, es decir, la dimensión vertical, la que ha acaparado todo el protagonismo: "Without the scales, the CEFR would have been largely ignored in European language education" (Alderson, 2007: 661). A este respecto, Figueras (2012) comenta que mientras que el uso de los niveles o escalas ha sido muy visible, el impacto de los descriptores de nivel ha sido menos obvio, aunque los descriptores hayan sido igualmente útiles. Igualmente, Barrault-Methy (2013) abunda en que las escalas han sido el acceso al Marco para numerosos países. Así, las escalas han sido el elemento más influyente del marco y, por ende, el más criticado.

Según Figueras (2012), las críticas al Marco se asocian con el contenido o bien con aspectos políticos.

En relación al aspecto político, Fulcher (2012) se refiere al hecho de que el Marco se haya institucionalizado y, en muchos casos, se emplee de manera preceptiva como barrera frente a la emigración o la movilidad de personas. Este uso normativo está muy alejado de la idea original del Marco, y sería inapropiado (North, 2007; 2014). Como indica Krumm (2007), los emigrantes constituyen un grupo heterogéneo completamente diferente al perfil de aprendiz con el que se concibió el marco, por lo que su uso estaría descontextualizado desde el punto de vista social y cultural. Dentro de esta perspectiva crítica, Deygers, Zeidler, Vilcu y Hamnes Carlsen (2017) añaden que algunos autores consideran que el marco se ha convertido en un instrumento de poder que impone una política de niveles en vez de considerar las necesidades del usuario o aprendiz.

En cuanto al contenido, se ha cuestionado, en primer lugar, la fundamentación del MCERL: la validez del marco con respecto a las teorías de adquisición de segundas lenguas (Little, 2007; North, 2007; Alderson, 2007; Hulstijn, 2007), el tratamiento desigual de las destrezas o la imposibilidad de abarcar todos los posibles niveles de cada escala (Alderson et al., 2006).

Además, se han señalado las deficiencias del Marco en el contexto de la evaluación, donde ejerce una gran influencia. A este respecto, se ha debatido la utilidad de los descriptores de niveles en la evaluación, puesto que no están redactados como objetivos y son muy generales, al no estar ligados a ningún idioma específico (Deygers et al., 2017). Finalmente, también se ha criticado que los niveles sean desiguales, ya que si se examinan las escalas 
detalladamente se pueden observar lagunas, solapamiento, ambigüedades e inconsistencias terminológicas. (Alderson et al., 2006; Alderson, 2007).

En realidad, las pretensiones de los autores del Marco eran muy modestas y gran parte de las críticas recibidas se deben a un uso incorrecto del texto. Los autores insisten en que su intención no era convertir el Marco en un documento normativo, y añaden que los descriptores se concibieron de manera general y que los niveles no fueron diseñados como escala interválica (Deygers et al., 2017). El Marco debía promover el debate sobre el aprendizaje de idiomas y servir de ayuda para la planificación curricular y las certificaciones lingüísticas (North, 2007). En este sentido, los autores del Marco sostienen que, pese las deficiencias señaladas, existe cierta uniformidad en el empleo de los descriptores y de los niveles por parte de las agencias evaluadoras como ALTE (Association of Language Testers in Europe) o EALTA (European Association for Language Testing and Assessment). Quince años después de su publicación, la relevancia del Marco se hace notar en la abundante literatura que ha inspirado y en la influencia que ha ejercido fuera de las fronteras europeas también (Barrault-Methy ,2013). Durante este período, como hemos apuntado anteriormente, ha sido objeto de numerosas críticas, pero también de alabanzas. Así, por ejemplo, se ha aplaudido el hecho que el Marco favorezca el diálogo (Deygers et al., 2017). Broek y Van den Ende añaden que: "Casi todos los aspectos del MCER son vulnerables a la crítica seria y, sin embargo, teniendo en cuenta la amplitud de su alcance, los profesionales de la lengua que lo han criticado por escrito son relativamente pocos en número" (2013: 5).

Así, el MCERL se ha convertido en un elemento imprescindible en el área del aprendizaje de idiomas superando con creces el alcance y la utilidad que sus creadores le confirieron. De esta suerte, los exámenes y certificados de dominio lingüístico en Europa han adaptado su sistema de puntuación al Marco o han sido reformulados de acuerdo con los descriptores (Deygers et al., 2017). Además, numerosos países e instituciones europeas han usado los niveles del Marco para fijar objetivos curriculares, requisitos para conseguir la ciudadanía, establecer requisitos de acceso o salida de la universidad, cuestión esta en la que se centra el presente estudio.

\subsection{La acreditación de idiomas en las universidades españolas.}

El proceso de globalización e internacionalización que vivimos a nivel mundial se deja sentir también en el ámbito de la educación y más en concreto, en la educación superior. En la universidad del siglo XXI el dominio de una lengua extranjera es una "necesidad transversal" (Fuentes González, 2013: 239).

En España, la acreditación de niveles de idioma cuenta con una relevancia fundamental en la actualidad, puesto que la implantación del EEES unido a la ampliación de políticas de acceso a la universidad (Bazo Martínez y González Álvarez, 2017) han sido determinantes para que gran parte del alumnado tenga que acreditar el conocimiento de una lengua extranjera para dar por finalizados sus estudios o tener acceso a los nuevos másteres (Fuentes González, 2013).

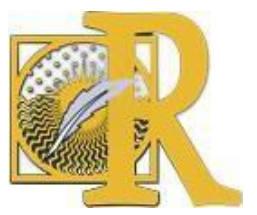


El impacto de este requisito en el acceso a determinados másteres y la percepción del alumnado ya fueron medidos en su momento (Raigón y Larrea, 2013).

Centrándonos en el ámbito de la acreditación para egresar, tema principal de este estudio, la mayoría de las universidades españolas exige a los estudiantes una acreditación lingüística con la cual se constata la competencia genérica "Conocimiento de una segunda lengua" según los principios del EEES. Aunque la redacción podría invitar a la confusión, "... el propósito principal de la acreditación lingüística en el seno de los nuevos grados y posgrados es constatar el dominio de una lengua extranjera" (Fuentes González, 2013: 248). Para conseguir esta acreditación, se ha hecho imprescindible el uso del MCERL, empleándose los niveles de referencia del marco para evaluar el dominio de la lengua.

En el caso de la acreditación lingüística, en España, la Conferencia de Rectores de Universidades Españolas (CRUE) determinó la creación de Mesas Lingüísticas en coordinación con la Asociación de Centros de Lenguas de Enseñanza Superior (ACLES) con el fin de "unificar criterios a la hora de acreditar niveles de conocimiento de diferentes lenguas extranjeras facilitando así la movilidad entre universidades españolas y su internacionalización" (ACLES, 2014: 9). Esto es, la intención final era consensuar criterios entre universidades y alcanzar acuerdos con respecto a la acreditación, y para ello resultaba imprescindible tratar el tema de los exámenes y certificaciones de nivel.

Pese a estas intenciones, con respecto a la acreditación de los estudiantes, a día de hoy, las universidades españolas no han consensuado la exigencia de nivel de lengua extranjera que se pide para egresar. Así, no en todas las universidades se exige un requisito lingüístico y, si se demanda, el nivel puede variar. En algunas comunidades, este tema ha sido regulado mediante normativas autonómicas; así, por ejemplo, en Andalucía se requiere un B1 para egresar en todas las universidades (Circular DGU-Junta de Andalucía 2010) mientras que en Cataluña se pide un B2 (DOGC 6551 de 30 de enero de 2014) según Bazo y González (2017).

Igualmente, los procedimientos para acreditar el nivel de dominio lingüístico varían enormemente ya que podemos encontrar: pruebas internas de una universidad, exámenes internacionales (Cambridge, TOEFL, etc.), pruebas CertAcles, estancias en el extranjero y realización de un número determinado de créditos en inglés. En el caso de la realización de exámenes o pruebas, el alumnado debe costear el importe de las mismas, aunque algunos gobiernos regionales y universidades han establecido ayudas económicas. Desde nuestro punto de vista $y$, aunque siempre han sido miradas con cierto recelo por su innegable elemento lucrativo, las acreditaciones son la herramienta más fiable y eficaz para medir la competencia lingüística en una lengua extranjera en este momento. 


\subsection{La acreditación de idiomas en la Universidad de Córdoba.}

En el caso que nos ocupa, la Universidad de Córdoba se rige por lo acordado entre todas las universidades públicas andaluzas en el "Convenio de Colaboración para la Acreditación de Lenguas Extranjeras" firmado el 2 de julio de 2011 y actualizado el 13 de junio de 2016 a instancias de la Comisión de Vigilancia del mismo. En él, se enumeran los certificados automáticamente válidos a estos efectos.

Dentro del respeto de la autonomía de cada una de las universidades, la UCO tiene la potestad de aceptar otros certificados siempre que cumplan los siguientes requisitos:

a) La certificación ha de ser emitida por una institución de reconocido prestigio.

b) Sólo las pruebas multi-competenciales pueden refrendar la competencia lingüística del alumnado, no pudiendo resultar en un certificado de acreditación las meras actividades formativas.

c) Tanto las destrezas productivas (oral y escrita) como las receptivas (oral y escrita) deberán de ser evaluadas y quedar reflejadas en el certificado que se aporte

Todos los alumnos universitarios en la región andaluza han de acreditar un nivel de competencia B1 en una lengua extranjera, quedando exentos los que cursen los grados en filologías de lenguas modernas o el grado de Traducción e Interpretación, según lo explicitado en el convenio arriba mencionado.

\section{Metodología.}

\subsection{Objetivos e hipótesis.}

El objetivo principal de este estudio es determinar qué idioma utiliza predominantemente el alumnado para el cumplimiento del requisito lingüístico. Asimismo, identificar si existe relación entre la nota de acceso y el nivel de acreditación.

Como objetivos secundarios de este estudio nos hemos planteado hallar el porcentaje de acreditaciones que acaparan las dos grandes empresas británicas del sector, Cambridge English Language Assessment y Trinity College London, comparándolo con el del propio centro de idiomas de la Universidad de Córdoba.

Se trata de un estudio predominantemente descriptivo en el que partimos de dos hipótesis principales:

1) la inmensa mayoría del alumnado utiliza el inglés para superar este requisito, contrariamente a los principios plurilingües establecidos en el MCERL.

2) los grados con nota de acceso más alta tienen un mayor número de alumnos que acreditan un nivel superior al exigido, es decir, B2 o más. 


\subsection{Variables y diseño.}

Las variables de este estudio son, por un lado, de carácter sociodemográfico o de identificación de la muestra en las que se encuadra el sexo o el grado que cursa el alumnado; y otras propias de la investigación como pueden ser el nivel de acreditación o el idioma utilizado.

EL diseño es de carácter descriptivo y ex post facto (Mateo, 2012), en el que los datos se obtendrán a posteriori de producirse el hecho.

\subsection{Muestra.}

Se ha analizado la información de todos los expedientes informados positivamente en el curso académico 2015/2016, lo que supone un total de 2185 expedientes, de los cuales 1221 corresponden a hombres y 964 mujeres. 2054 han utilizado el inglés como idioma para acreditar. El resto se divide entre francés (63), italiano (60) y otros idiomas (8). 1823 estudiantes han acreditado nivel B1, 313 B2, 45 C1 y 4 nivel C2.

El alumnado puede presentar su acreditación en cualquier momento:

\begin{tabular}{l|l|l} 
CURSO & FRECUENCIA & PORCENTAJE \\
\hline PRIMERO & 163 & $7,5 \%$ \\
\hline SEGUNDO & 188 & $8,6 \%$ \\
\hline TERCERO & 227 & $10,4 \%$ \\
\hline CUARTO & 1404 & $64,3 \%$ \\
\hline QUINTO & 203 & $9,3 \%$ \\
\hline TOTAL & 2185 & $100 \%$
\end{tabular}

Tabla 1. Curso de acreditación por titulación.

Los estudiantes han de tramitar sus expedientes a través de la sede electrónica de la UCO siguiendo un procedimiento específico habilitado a tal efecto, al que han de adjuntar una copia digitalizada de su certificado de acreditación lingüística. Además de su información personal, los expedientes contienen información relativa a sus estudios, nivel de acreditación, curso en el que están matriculados...

\subsection{Estrategias.}

Para el presente trabajo, hemos utilizado el paquete estadístico SPSS (v.18); en primer lugar, hemos realizado un estudio descriptivo. A este respecto, hemos llevado a cabo tablas de contingencia utilizando diferentes combinaciones de variables.

En segundo lugar, hemos realizado análisis de varianzas para comprobar si existe una relación probada entre determinadas variables y el nivel de acreditación, como los resultados del estudio descriptivo parecen indicar. Dentro de los análisis de varianzas, trabajaremos para establecer relaciones entre los niveles de acreditación y las variables "sexo", "nota de acceso" y "macroárea". 


\section{Resultados.}

\subsection{Descriptivo.}

Este apartado se divide en cinco análisis con datos relativos al idioma y nivel con el que supera el requisito, a la acreditación utilizada, al nivel de acreditación en relación con la titulación y en relación con el área y, finalmente, al momento en el que el alumnado incorpora este requisito a su expediente.

\begin{tabular}{|c|c|c|c|c|c|c|c|}
\hline \multirow{2}{*}{\multicolumn{3}{|c|}{ IDIOMA DE ACREDITACIÓN }} & \multicolumn{4}{|l|}{ | NIVEL } & \multirow[b]{2}{*}{ Total } \\
\hline & & & \begin{tabular}{|l|} 
B1 \\
54
\end{tabular} & \begin{tabular}{|l} 
B2 \\
7
\end{tabular} & C1 & $\mathrm{C} 2$ & \\
\hline & Francés & $\begin{array}{l}\text { Recuento } \\
\% \text { dentro de Idioma } \\
\% \text { del total }\end{array}$ & $\begin{array}{l}54 \\
85,7 \% \\
2,5 \%\end{array}$ & $\begin{array}{l}7 \\
11,1 \% \\
0,3 \%\end{array}$ & $\begin{array}{l}2 \\
3,2 \% \\
0,1 \%\end{array}$ & $\begin{array}{l}0 \\
0,0 \% \\
0,0 \%\end{array}$ & $\begin{array}{l}63 \\
100,0 \% \\
2,9 \%\end{array}$ \\
\hline & Inglés & $\begin{array}{l}\text { Recuento } \\
\% \text { dentro de Idioma } \\
\% \text { del total }\end{array}$ & $\begin{array}{l}1706 \\
83,1 \% \\
78,1 \%\end{array}$ & $\begin{array}{l}303 \\
14,8 \% \\
13,9 \%\end{array}$ & $\begin{array}{l}42 \\
2,0 \% \\
1,9 \%\end{array}$ & $\begin{array}{l}3 \\
0,1 \% \\
0,1 \%\end{array}$ & $\begin{array}{l}2054 \\
100,0 \% \\
94,0 \%\end{array}$ \\
\hline & Italiano & $\begin{array}{l}\text { Recuento } \\
\% \text { dentro de Idioma } \\
\% \text { del total }\end{array}$ & \begin{tabular}{|l|}
57 \\
$95,0 \%$ \\
$2,6 \%$
\end{tabular} & $\begin{array}{l}3 \\
5,0 \% \\
0,1 \%\end{array}$ & $\begin{array}{l}0 \\
0,0 \% \\
0,0 \%\end{array}$ & $\begin{array}{l}0 \\
0,0 \% \\
0,0 \%\end{array}$ & $\begin{array}{l}60 \\
100,0 \% \\
2,7 \%\end{array}$ \\
\hline & Otro & $\begin{array}{l}\text { Recuento } \\
\% \text { dentro de Idioma } \\
\% \text { del total }\end{array}$ & $\begin{array}{l}6 \\
75,0 \% \\
0,3 \%\end{array}$ & $\begin{array}{l}0 \\
0,0 \% \\
0,0 \%\end{array}$ & $\begin{array}{l}1 \\
12,5 \% \\
0,0 \%\end{array}$ & $\begin{array}{l}1 \\
12,5 \% \\
0,0 \%\end{array}$ & $\begin{array}{l}8 \\
100,0 \% \\
0,4 \%\end{array}$ \\
\hline Total & & $\begin{array}{l}\text { Recuento } \\
\% \text { del total }\end{array}$ & \begin{tabular}{|l|}
1823 \\
$83,4 \%$
\end{tabular} & $\begin{array}{l}313 \\
14,3 \%\end{array}$ & \begin{tabular}{|l|}
45 \\
$2,1 \%$
\end{tabular} & \begin{tabular}{|l|}
4 \\
$0,2 \%$
\end{tabular} & \begin{tabular}{|l|}
2185 \\
$100,0 \%$
\end{tabular} \\
\hline
\end{tabular}

Tabla 2. Acreditaciones por nivel e idioma.

En un primer acercamiento a los datos, podemos observar que, del total de 2185 expedientes, 2054 corresponden a inglés, lo que supone un $94 \%$ de todos los expedientes tramitados. Seguidos por este idioma, aparece francés, con 2,9\% de expedientes e italiano, con $2,7 \%$. La categoría "otro" representa un residual $0,4 \%$, con tan sólo 8 casos.

En cuanto al nivel, el B1, mínimo para cumplir el requisito, supone el $83,4 \%$ del total de los casos. 


\begin{tabular}{l|l|l} 
ACREDITACIONES & FRECUENCIA & $\%$ \\
\hline TRINITY COLLEGE: INTEGRATED SKILLS IN ENGLISH (ISE) & 988 & $45,2 \%$ \\
\hline CAMBRIDGE EXAMINATIONS & 774 & $35,4 \%$ \\
\hline UCOIDIOMAS & 220 & $10,1 \%$ \\
\hline ACLES & 56 & $2,6 \%$ \\
\hline ESCUELA OFICIAL DE IDIOMAS & 56 & $2,6 \%$ \\
\hline DELF & 23 & $1,1 \%$ \\
\hline PROGETTO LINGUA ITALIANA DANTE ALIGHIERI (PLIDA) & 22 & $1 \%$ \\
\hline OTRAS & 14 & $0,6 \%$ \\
\hline ALLIANCE FRANÇAISE & 9 & $0,4 \%$ \\
\hline UNIVERSIDADES ANDALUZAS & 6 & $0,3 \%$ \\
\hline CERTIFICATO DI ITALIANO COME LINGUA STRANIERA & 5 & $0,2 \%$ \\
(CILS) & & \\
\hline IELTS & 4 & $0,2 \%$ \\
\hline APTIS & 4 & $0,2 \%$ \\
\hline TEST DE CONNAISANCE DE FRANÇAIS (TCF) & 2 & $0,1 \%$ \\
\hline CERTIFICATO DI CONOSCENZA DELLA LINGUA ITALIANA \\
(CELI) & 1 & $0,04 \%$ \\
\hline TOEFL & 1 & \\
\hline TOTAL & 2185 & 100
\end{tabular}

Tabla 3. Acreditaciones por instituciones.

Las grandes instituciones británicas de acreditación (Trinity y Cambridge) acaparan más del $80 \%$ de certificados aportados por los alumnos. Si sólo tenemos en cuenta los certificados en lengua inglesa, estas empresas copan el $85,7 \%$ del total. Fuera de ellas, la otra institución con cierta representatividad es Ucoidiomas (Servicio de Idiomas de la propia universidad), con su certificado propio (10,1\% entre todos los idiomas que acredita esta entidad). 


\section{INTERNATIONAL JOURNAL OF EDUCATIONAL \\ RESEARCH AND INNOVATION \\ REVISTA INTERNACIONAL DE INVESTIGACIÓN \\ EINNOVACIÓN EDUCATIVA}

Antonio Raigón \& Ángela Larrea. La acreditación de idiomas en las universidades españolas: Un estudio cuantitativo en la Universidad de Córdoba

\begin{tabular}{|c|c|c|c|c|c|c|}
\hline \multirow{2}{*}{ TITULACIONES } & & \multicolumn{4}{|l|}{ NIVEL } & \multirow{2}{*}{$\begin{array}{l}\text { NOTA } \\
\text { ACCESO }\end{array}$} \\
\hline & & B1 & B2 & $\mathrm{C} 1$ & $\mathrm{C} 2$ & \\
\hline \multirow{2}{*}{$\begin{array}{l}\text { Doble Grado de Derecho y Administración y } \\
\text { Dirección de Empresas }\end{array}$} & Recuento & 79 & 24 & 4 & 0 & \multirow[t]{2}{*}{6,916} \\
\hline & \% Titulación & $73,8 \%$ & $22,4 \%$ & $3,7 \%$ & $0,0 \%$ & \\
\hline \multirow{2}{*}{$\begin{array}{l}\text { Grado de Administración y Dirección de } \\
\text { Empresas }\end{array}$} & Recuento & 111 & 19 & 1 & 0 & \multirow[t]{2}{*}{8,201} \\
\hline & \% Titulación & $84,7 \%$ & $14,5 \%$ & $0,8 \%$ & $0,0 \%$ & \\
\hline \multirow[t]{2}{*}{ Grado de Biología } & Recuento & 90 & 8 & 3 & 0 & \multirow[t]{2}{*}{7,115} \\
\hline & \% Titulación & $89,1 \%$ & $7,9 \%$ & $3,0 \%$ & $0,0 \%$ & \\
\hline \multirow[t]{2}{*}{ Grado de Bioquímica } & Recuento & 31 & 12 & 1 & 0 & \multirow[t]{2}{*}{11,561} \\
\hline & \% Titulación & $70,5 \%$ & $27,3 \%$ & $2,3 \%$ & $0,0 \%$ & \\
\hline \multirow{2}{*}{$\begin{array}{l}\text { Grado de Ciencia y Tecnología de los } \\
\text { Alimentos }\end{array}$} & Recuento & 40 & 4 & 0 & 0 & \multirow[t]{2}{*}{7,466} \\
\hline & \% Titulación & $90,9 \%$ & $9,1 \%$ & $0,0 \%$ & $0,0 \%$ & \\
\hline \multirow[t]{2}{*}{ Grado de Ciencias Ambientales } & Recuento & 21 & 2 & 0 & 0 & \multirow[t]{2}{*}{5,000} \\
\hline & \% Titulación & $91,3 \%$ & $8,7 \%$ & $0,0 \%$ & $0,0 \%$ & \\
\hline \multirow[t]{2}{*}{ Grado de Derecho } & Recuento & 113 & 14 & 3 & 1 & \multirow[t]{2}{*}{5,120} \\
\hline & \% Titulación & $86,3 \%$ & $10,7 \%$ & $2,3 \%$ & $0,8 \%$ & \\
\hline \multirow[t]{2}{*}{ Grado de Educación Infantil } & Recuento & 212 & 11 & 0 & 0 & \multirow[t]{2}{*}{5,902} \\
\hline & \% Titulación & $95,1 \%$ & $4,9 \%$ & $0,0 \%$ & $0,0 \%$ & \\
\hline \multirow[t]{2}{*}{ Grado de Educación Primaria } & Recuento & 215 & 32 & 2 & 0 & \multirow[t]{2}{*}{6,528} \\
\hline & \% Titulación & $86,3 \%$ & $12,9 \%$ & $0,8 \%$ & $0,0 \%$ & \\
\hline \multirow[t]{2}{*}{ Grado de Física } & Recuento & 17 & 10 & 3 & 1 & \multirow[t]{2}{*}{5,000} \\
\hline & \% Titulación & $54,8 \%$ & $32,3 \%$ & $9,7 \%$ & $3,2 \%$ & \\
\hline \multirow[t]{2}{*}{ Grado de Historia } & Recuento & 31 & 4 & 1 & 0 & \multirow[t]{2}{*}{5,000} \\
\hline & \% Titulación & $86,1 \%$ & $11,1 \%$ & $2,8 \%$ & $0,0 \%$ & \\
\hline \multirow[t]{2}{*}{ Grado de Historia del Arte } & Recuento & 35 & 5 & 0 & 0 & \multirow[t]{2}{*}{5,000} \\
\hline & \% Titulación & $87,5 \%$ & $12,5 \%$ & $0,0 \%$ & $0,0 \%$ & \\
\hline \multirow{2}{*}{$\begin{array}{l}\text { Grado de Ingeniería Agroalimentaria y del } \\
\text { Medio Rural }\end{array}$} & Recuento & 44 & 8 & 1 & 0 & 5,000 \\
\hline & \% Titulación & $83,0 \%$ & $15,1 \%$ & $1,9 \%$ & $0,0 \%$ & \\
\hline Grado de Ingeniería Civil & Recuento & 72 & 9 & 1 & 0 & 5,000 \\
\hline & \% Titulación & $87,8 \%$ & $11,0 \%$ & $1,2 \%$ & $0,0 \%$ & \\
\hline Grado de Ingeniería de Recursos Energéticos & Recuento & 12 & 0 & 0 & 0 & 5,000 \\
\hline y Mine & \% Titulación & $100,0 \%$ & $0,0 \%$ & $0,0 \%$ & $0,0 \%$ & \\
\hline Grado de Ingeniería Eléctrica & Recuento & 23 & 5 & 2 & 0 & 5,000 \\
\hline & \% Titulación & $76,7 \%$ & $16,7 \%$ & $6,7 \%$ & $0,0 \%$ & \\
\hline Grado de Ingeniería Electrónica Industrial & Recuento & 36 & 10 & 1 & 0 & 5,000 \\
\hline & \% Titulación & $76,6 \%$ & $21,3 \%$ & $2,1 \%$ & $0,0 \%$ & \\
\hline
\end{tabular}




\begin{tabular}{l|l|llll|l}
\hline Grado de Ingeniería Forestal & Recuento & 35 & 3 & 0 & 0 & 5,000 \\
& $\%$ Titulación & $92,1 \%$ & $7,9 \%$ & $0,0 \%$ & $0,0 \%$ & \\
\hline Grado de Ingeniería Informática & Recuento & 61 & 11 & 0 & 1 & 5,670 \\
& $\%$ Titulación & $83,6 \%$ & $15,1 \%$ & $0,0 \%$ & $1,4 \%$ & \\
\hline Grado de Ingeniería Mecánica & Recuento & 68 & 12 & 0 & 0 & 5,000 \\
& $\%$ Titulación & $85,0 \%$ & $15,0 \%$ & $0,0 \%$ & $0,0 \%$ & \\
\hline Grado de Medicina & Recuento & 90 & 52 & 12 & 0 & 12,569 \\
& $\%$ Titulación & $58,4 \%$ & $33,8 \%$ & $7,8 \%$ & $0,0 \%$ & \\
\hline Grado de Química & Recuento & 46 & 3 & 2 & 0 & 5,000 \\
& $\%$ Titulación & $90,2 \%$ & $5,9 \%$ & $3,9 \%$ & $0,0 \%$ & \\
\hline Grado de Relaciones Laborales y Recursos & Recuento & 86 & 5 & 0 & 0 & 5,000 \\
Humanos & $\%$ Titulación & $94,5 \%$ & $5,5 \%$ & $0,0 \%$ & $0,0 \%$ & \\
\hline Grado de Turismo & Recuento & 33 & 8 & 0 & 0 & 5,000 \\
& $\%$ Titulación & $80,5 \%$ & $19,5 \%$ & $0,0 \%$ & $0,0 \%$ & \\
\hline Grado de Veterinaria & Recuento & 95 & 17 & 5 & 0 & 11,206 \\
& $\%$ Titulación & $81,2 \%$ & $14,5 \%$ & $4,3 \%$ & $0,0 \%$ & \\
\hline Graduado en Enfermería & Recuento & 121 & 18 & 2 & 1 & 10,550
\end{tabular}

Tabla 4. Nivel de acreditación por titulación.

Como se puede observar, las titulaciones con niveles más altos de acreditación son Física, Medicina, Bioquímica y el doble grado de Derecho y ADE. Medicina y Bioquímica, tienen las dos notas más altas de acceso de la UCO.

Las titulaciones con mayor tanto por ciento de acreditados con nivel B1 son Ingeniería de Recursos Mineros, Educación Infantil y Relaciones Laborales, con notas de acceso mínimas en dos de los casos y de 5,9 en Ed. Infantil.

Realizando una comparación análoga a la anterior, pero agrupando por áreas, podemos observar que tan sólo el área de Ciencias de la Salud se encuentra muy por debajo de la media de acreditaciones de B1, es decir, su alumnado presenta un mayor número de acreditaciones de B2 y superior (entre 6 y 10 puntos porcentuales más que otras áreas). Se da la circunstancia de que los grados incluidos en Ciencias de la Salud (Veterinaria, Medicina y Enfermería) cuentan con 3 de las 4 notas de acceso más altas de la Universidad. 


\begin{tabular}{|c|c|c|c|c|c|c|}
\hline & & B1 & B2 & $\mathrm{C} 1$ & $\mathrm{C} 2$ & \\
\hline \multirow[t]{2}{*}{ Artes y Humanidades } & Recuento & 68 & 9 & 1 & 0 & 78 \\
\hline & \% dentro de Áreas & $87,2 \%$ & $11,5 \%$ & $1,3 \%$ & $0,0 \%$ & $100,0 \%$ \\
\hline \multirow[t]{2}{*}{ Ciencias } & Recuento & 245 & 39 & 10 & 1 & 295 \\
\hline & \% dentro de Áreas & $83,1 \%$ & $13,2 \%$ & $3,4 \%$ & $0,3 \%$ & $100,0 \%$ \\
\hline \multirow{2}{*}{ Ciencias de la Salud } & Recuento & 307 & 91 & 19 & 1 & 418 \\
\hline & \% dentro de Áreas & $73,4 \%$ & $21,8 \%$ & $4,5 \%$ & $0,2 \%$ & $100,0 \%$ \\
\hline \multirow[t]{2}{*}{ Ingeniería y Arquitectura } & Recuento & 352 & 61 & 5 & 1 & 419 \\
\hline & \% dentro de Áreas & $84,0 \%$ & $14,6 \%$ & $1,2 \%$ & $0,2 \%$ & $100,0 \%$ \\
\hline \multirow[t]{2}{*}{ Sociales y Jurídicas } & Recuento & 851 & 113 & 10 & 1 & 975 \\
\hline & \% dentro de Áreas & $87,3 \%$ & $11,6 \%$ & $1,0 \%$ & $0,1 \%$ & $100,0 \%$ \\
\hline \multirow[b]{2}{*}{ Total } & Recuento & 1823 & 313 & 45 & 4 & 2185 \\
\hline & $\%$ del total & $83,4 \%$ & $14,3 \%$ & $2,1 \%$ & $0,2 \%$ & $100,0 \%$ \\
\hline
\end{tabular}

Tabla 5. Niveles de acreditación por Macroárea.

Como se puede ver en la tabla 1, los alumnos de la UCO en su mayoría esperan al último curso para acreditar su nivel de competencia lingüística. 1404 lo hacen en cuarto, que es el último curso para todas las titulaciones excepto Medicina, Veterinaria, y los dos dobles grados. Deduciendo estos alumnos, el total que acredita en cuarto es 1370 . Si sumamos los 203 que lo hacen en quinto curso (sólo de los grados arriba mencionados), tenemos un total de 1573 , que son el $72 \%$ de todos los expedientes analizados. Esto supone que entre primero y segundo, sólo el $16,1 \%$ de los alumnos formaliza el trámite de la inclusión del B1 en su expediente académico.

\subsection{Inferencial.}

A continuación, presentamos los resultados obtenidos en los tres análisis de varianzas realizados.

Hemos categorizado las notas de acceso con el objeto de efectuar una Anova, a la cual se le aplicado previamente el Test de Levene, que ha reflejado homogeneidad en las varianzas, obteniendo los siguientes datos distintivos que se exponen en la siguiente tabla. 


\begin{tabular}{l|l|l|l|l} 
Grupo & $\mathrm{N}$ & Media & $\mathrm{s}$ & $\mathrm{F}$ y $\mathrm{p}$ \\
\hline 1 & 1170 & 1,14 & 0,408 & F=24,307 y $\mathrm{p}=0.000$ \\
\hline 2 & 553 & 1,18 & 0,425 & favorable a \\
\hline 3 & 462 & 1,32 & 0,562 & Grupo 3>Grupo 1 \\
\hline TOTAL & 2185 & 1,19 & 0,454 & Grupo 3>Grupo 2
\end{tabular}

Tabla 6. Anova de la nota de acceso en relación con el nivel de acreditación.

Los participantes con nota de acceso superior (grupo 3, lo cuales equivalen a mayor que $8,2011)$ posee un mayor nivel de acreditación, $F(2,2185)=24,307, p=0,000, \eta=0,09$ que el grupo con nota de acceso más baja (grupo 1, nota menor que 5,9020), $t(2185)=6,88$ y $p=0,000$. Asimismo, el grupo 3 también puntúa más alto en referencia al nivel de acreditación que el 2 (notas de acceso entre 5,9021-8,2010), t(2185)=4,821 y $\mathrm{p}=0,000$.

Teniendo en cuenta la variable de pertenencia a grados de diferentes ramas de conocimiento, hemos efectuado una Anova, a la cual se le aplicado previamente el Test de Levene que ha reflejado homogeneidad en las varianzas, obteniendo los siguientes datos distintivos que se exponen en la siguiente tabla.

\begin{tabular}{l|l|l|l|l} 
Macroárea & $\mathrm{N}$ & Media & $\mathrm{S}$ & $\mathrm{F}$ y $\mathrm{p}$ \\
\hline Artes y Humanidades & 78 & 1,14 & 0,386 & $\mathrm{~F}=11,729$ y $\mathrm{p}=0.000$ \\
\cline { 1 - 2 } Ciencias & 295 & 1,21 & 0,505 & favorable a \\
\cline { 1 - 2 } Ciencias de la Salud & 418 & 1,32 & 0,568 & CC. Salud $>$ Artes y Hum. \\
\cline { 1 - 2 } Ingeniería y Arquitectura & 419 & 1,18 & 0,429 & CC. Salud $>$ Ciencias \\
\cline { 1 - 2 } Sociales y Jurídicas & 975 & 1,14 & 0,383 & CC. Salud $>$ Ing. y Arq. \\
\cline { 1 - 2 } Total & 2185 & 1,19 & 0,454 & CC. Salud $>$ Soc. y Jur.
\end{tabular}

Tabla 7. Anova del nivel de acreditación en relación a las macroáreas.

Los alumnos del área de Ciencias de la Salud acreditan un mayor nivel de competencia lingüística que el resto del alumnado de la Universidad de Córdoba, $F(4,2180)=11,729$, $\mathrm{p}=0,000, \eta 2=0,022$. Esto se puede observar en relación al alumnado de Artes y Humanidades, $\mathrm{t}(2180)=3,1818$ y $\mathrm{p}=0,016$; al de Ciencias, $\mathrm{t}(2180)=3,118$ y $\mathrm{p}=0,020$; al de Ingeniería y Arquitectura, $\mathrm{t}(2180)=4,4839$ y $\mathrm{p}=0,000 ; \mathrm{y}$, finalmente, al de Sociales y Jurídicas, $\mathrm{t}(2180)=6,769$ y $\mathrm{p}=0,000$. Estos resultados vienen a refrendar los apuntados en el estudio descriptivo.

\begin{tabular}{l|l|l|l|l} 
Sexo & $\mathrm{N}$ & Media & $\mathrm{S}$ & T y $\mathrm{p}$ \\
\hline Mujer & 1221 & 1,17 & 0,426 & T=-2,612 y $\mathrm{p}=0.009$ \\
Hombre & 964 & 1,22 & 0,485 & a favor de hombre
\end{tabular}

Tabla 8. T-Student del nivel de acreditación teniendo en cuenta la variable sexo.

Los hombres que participan en este estudio han acreditado un mayor nivel de acreditación lingüística ( $\mathrm{t}=-2,612$ y $\left.\mathrm{p}=0.009,{ }^{-}{ }^{-22}\right)$.

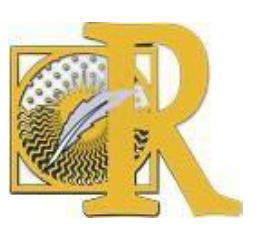




\section{Discusión de resultados y conclusiones.}

Si observamos los resultados en relación con la primera hipótesis, la omnipresencia del inglés como lengua de acreditación en nuestra institución, los resultados no pueden ser más claros ni más contrarios al discurso del Consejo de Europa (2001: 134), máximo organismo en materia de política lingüística común. El hecho de que 19 de cada 20 alumnos de la UCO acrediten con el inglés su segundo idioma prueba que el discurso plurilingüe de las instituciones no ha calado en la comunidad educativa. En nuestra opinión, sólo variables tan aleatorias como estancias Erasmus en Italia o la presencia de institutos de educación secundaria bilingües de francés en la provincia de Córdoba pueden hacer que el número de acreditados en inglés no sea aún mayor.

Este fenómeno, que seguramente sea análogo en el resto del territorio nacional, hace que el negocio de las acreditaciones lingüísticas tenga un gran impacto. En este sentido, las grandes compañías británicas de acreditación disfrutan de una amplísima cuota de mercado: 4 de cada 5 alumnos en la UCO las utilizan para acreditar su nivel de idioma.

Los esfuerzos de los modelos públicos (Escuelas Oficiales de Idiomas, ACLES o Servicios de Idiomas propios de cada universidad) no parecen estar dando respuesta a esta demanda. Las razones de esta notoria falta de interés por opciones mucho más económicas para el alumnado pueden proporcionar una futura línea de investigación. Lo que resulta innegable es que tan sólo el 15,6\% del alumnado opta por una acreditación emitida por una institución pública. Este hecho hace que los Servicios de Idiomas de las Universidades Públicas ofrezcan, además de su propio certificado, un amplio abanico de posibilidades entre las que se encuentran Trinity, TOEFL y Cambridge, por ejemplo. (http://www.uco.es/idiomas/acreditaciones-oficiales-idiomas/).

Para nuestra segunda hipótesis, la referida a la relación de la nota de acceso con el nivel de acreditación, las tablas de contingencia efectuadas han arrojado los porcentajes de los niveles de acreditación entre los alumnos de distintos grados (y macroáreas). En concreto, de los tres grados con niveles de acreditación mayor, dos de ellos (Medicina y Bioquímica) tienen la nota de acceso más alta de la UCO $(12,569$ y 11,561). Sin embargo, entre las tres primeras con más acreditados se encuentra Física, que tiene una nota mínima de acceso (5). Las tres titulaciones con nivel más bajo de acreditación tienen notas de acceso bajas (dos de ellas 5 y otra 5,9). El análisis de varianzas ejecutado nos ha permitido corroborar nuestra segunda hipótesis, demostrando que existe una incidencia entre la nota de acceso y un mayor nivel de competencia lingüística acreditada.

De igual manera, hemos podido constatar que el alumnado de Ciencias de la Salud es el que presenta un nivel de acreditación de idioma más alto en la Universidad de Córdoba. Las titulaciones que lo componen (Medicina, Enfermería y Veterinaria) también tienen en común una nota de acceso muy alta. Parece confirmarse así que el desarrollo de la competencia lingüística está unido al desarrollo de otras competencias o, en cualquier caso, al grado de realización académica. De manera complementaria, sería interesante hacer un estudio en el que los factores socioeconómicos pudiesen relacionarse con la nota de acceso.

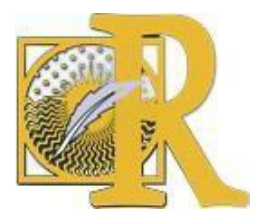


Por otro lado, hemos de significar que los varones acreditan un nivel de idioma más alto que las mujeres en la UCO. Finalmente, podemos constatar que el alumnado espera hasta su último año para cumplir un requisito que conocen y pueden aportar desde el primer día que ingresan en la universidad. Esto nos hace pensar que la formación lingüística de los alumnos en educación secundaria aún no ha tenido en cuenta este nuevo condicionante. Puesto que muchos investigadores han resaltado el bajo desarrollo de las destrezas orales entre los estudiantes españoles (Lagasabaster, 2008; Corpas y Madrid, 2009; Cortina, 2011), quizás haya llegado el momento de cuestionarnos por qué los resultados son tan decepcionantes, máxime cuando la mayoría del alumnado pasa al menos diez años en un aula de lengua extranjera antes de llegar a la universidad.

Sin embargo, no hemos de descartar la tendencia alcista del nivel de competencia lingüística acreditado por el alumnado, que se deberá concretar en un estudio futuro que nos permita cuantificar esta mejora en el tiempo. Si este hecho se constatara, podría indicar la efectividad del requisito lingüístico como método de reactivación de la enseñanza de segundas lenguas en todas las etapas educativas.

\section{Bibliografía.}

- ACLES (2014). CertaAcles. Modelo de Acreditación de los exámenes de ACLES. Recuperado de: http://www.acles.es/multimedia/enlaces/9/files/fichero 136.pdf

- Alderson, J. (2007). The CEFR and the need for more research. The Modern Language Journal, 91(4), 659-663.

- Alderson, J., Figueras, N., Kuijper, H., Nold, G., Takala, S., \& Tardieu, C. (2006). Analysing tests of reading and listening in relation to the common European framework of reference: The experience of the Dutch CEFR construct project. Language Assessment Quarterly: An International Journal, 3(1), 3-30.

- Barrault-Méthy, A. M. (2013). The Common European Framework of Reference: The globalisation of language education policy. English Language Teaching Journal 67(4), 500-502.

- Bazo Martínez, P. \& González Álvarez, D. (2017). Política Lingüística para la Internacionalización del Sistema Universitario Español. Madrid: CRUE.

- Broek, S. \& Van den Ende, I. (2013). La aplicación del Marco Común Europeo de Referencia para las Lenguas en los sistemas educativos europeos. Bruselas: Parlamento Europeo.

- Clouet, R. (2010). El enfoque del Marco Común Europeo de Referencia para las Lenguas: Unas reflexiones sobre su puesta en práctica en las facultades de traducción e interpretación en España. Revista de Lingüística Teórica y Aplicada, 48(2), 71-92.

- Corpas, Ma D. \& Daniel Madrid, D. (2009). Desarrollo de la comprensión oral en inglés como LE al término de la Educación Secundaria Obligatoria española. Porta Linguarum, $11,129-145$.

- Cortina, B. (2011). La expresión oral en lengua inglesa de los futuros maestros: un reto para el Espacio Europeo de Educación Superior. Porta Linguarum, 16, 155-177.

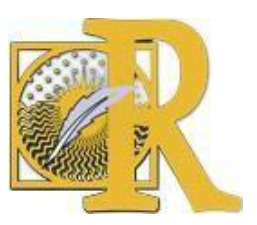

Fecha de recepción: 26-11-2018 Fecha de aceptación: 03-06-2019

Raigón-Rodríguez, A. \& Larrea-Espinar, A. (2019). La acreditación de idiomas en las universidades españolas: Un estudio cuantitativo en la Universidad de Córdoba 
- Council of Europe (1975). The Threshold Level. Strasbourg: Council of Europe.

- Council of Europe (1992). Transparency and coherence in language learning in Europe: Objectives, evaluation, certification. Report on the Rüschlikon Symposium. Strasbourg: Council of Europe.

- Deygers, B., Zeidler, B., Vilcuc, D. \& Hamnes Carlsend, C. (2017). One Framework to Unite Them All? Use of the CEFR in European University Entrance Policies. Language Assessment Quarterly, 9, 1-13.

- European Commission (2012). Special Eurobarometer 386 - Wave EB77.1, Europeans and Languages. Brussels: European Comission.

- Figueras, N. (2012). The impact of the CEFR. ELT Journal, 66(4), 477-485.

- Fuentes González, A. D. (2013). De momento, lenguas alla bolognese. Porta Linguarum, 19, 239-256.

- Fulcher, G. (2012). Scoring performance tests, en Fulcher, G. \& Davidson, F. (Eds.). The Routledge handbook of language testing (pp.378-392). London: Routledge.

- Hulstijn, J. (2007). The shaky ground beneath the CEFR: Quantitative and qualitative dimensions of language proficiency. The Modern Language Journal, 91(4), 663-667.

- Krumm, H. J. (2007). Profiles instead of levels: The CEFR and its (ab)uses in the context of migration. Modern Language Journal, 91(4) 667-669.

- Lasagabaster, D. (2008). Foreign language competence in content and language integrated courses. The Open Applied Linguistics Journal, 1, 30-41.

- Little, D. (2007). The common European framework of reference for languages: Perspectives on the making of supranational language education policy. The Modern Language Journal, 91(4), 645-655.

- Little, D. (2011). The Common European Framework of Reference for Languages, the European Language Portfolio, and language learning in higher education. CercleS 2011, $1(1), 1-21$.

- Mateo, J. (2012). La investigación ex post-facto. En R. Bisquerra (coord.). Metodología de la Investigación Educativa (pp.195-229). Madrid: La Muralla.

- North, B. (2007). The CEFR illustrative descriptor scales. The Modern Language Journal, 91(4), 656-659.

- North, B. (2014). Putting the common European framework of reference to good use. Language Teaching, 47(2), 228-249.

- Raigón Rodríguez, A. y Larrea Espinar, A. (2013). Análisis y percepción por parte del alumnado del requisito lingüístico en el acceso al Máster de Formación del Profesorado de Educación Secundaria. Porta Linguarum, 20, 63-76.

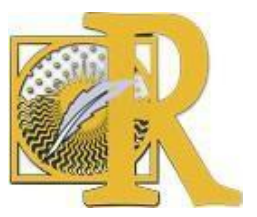

Fecha de recepción: 26-11-2018 Fecha de aceptación: 03-06-2019 